\title{
Thermoelectric Characterization of Electronic Properties of GaMnAs Nanowires
}

\author{
Phillip M. Wu, ${ }^{1}$ Waldomiro Paschoal Jr., ${ }^{1,2}$ Sandeep Kumar, ${ }^{1}$ \\ Christian Borschel, ${ }^{3}$ Carsten Ronning, ${ }^{3}$ Carlo M. Canali, ${ }^{4}$ \\ Lars Samuelson, ${ }^{1}$ Håkan Pettersson, ${ }^{1,2}$ and Heiner Linke ${ }^{1}$ \\ ${ }^{1}$ Division of Solid State Physics and The Nanometer Structure Consortium (nmC@LU), Lund University, \\ P.O. Box 118, 22100 Lund, Sweden \\ ${ }^{2}$ Department of Mathematics, Physics, and Electrical Engineering, Halmstad University, P.O. Box 823, 30118 Halmstad, Sweden \\ ${ }^{3}$ Institute for Solid State Physics, Jena University, Max-Wien-Platz 1, 07743 Jena, Germany \\ ${ }^{4}$ Division of Physics, School of Computer Science, Physics and Mathematics, Linnoeus University, 39233 Kalmar, Sweden
}

Correspondence should be addressed to Phillip M. Wu, phillip.wu@ftf.lth.se

Received 21 June 2012; Accepted 23 August 2012

Academic Editor: Magnus T. Borgstrom

Copyright (c) 2012 Phillip M. Wu et al. This is an open access article distributed under the Creative Commons Attribution License, which permits unrestricted use, distribution, and reproduction in any medium, provided the original work is properly cited.

Nanowires with magnetic doping centers are an exciting candidate for the study of spin physics and proof-of-principle spintronics devices. The required heavy doping can be expected to have a significant impact on the nanowires' electron transport properties. Here, we use thermopower and conductance measurements for transport characterization of $\mathrm{Ga}_{0.95} \mathrm{Mn}_{0.05} \mathrm{As}_{\text {nanowires over a }}$ broad temperature range. We determine the carrier type (holes) and concentration and find a sharp increase of the thermopower below temperatures of $120 \mathrm{~K}$ that can be qualitatively described by a hopping conduction model. However, the unusually large thermopower suggests that additional mechanisms must be considered as well.

\section{Introduction}

Self-assembled semiconducting epitaxial nanowires are promising building blocks for field effect transistors [1], sensors [2], and solar cells [3]. An exciting new direction, which has recently been shown to be possible due to successful incorporation of magnetic Mn dopants into epitaxially grown GaAs nanowires (NWs) [4-11], is their use for proofof-concept spintronics devices [12]. The doping techniques are advancing rapidly, and it has recently been shown that ion beam implantation can produce single crystalline, homogeneously doped GaMnAs NWs [13]. Furthermore, a recent study found that the Curie temperature of GaMnAs nanostrips could be enhanced to $200 \mathrm{~K}$ with nanostructure engineering [14], suggesting the possibility for nanowirebased devices to operate at higher temperatures compared to thin films or bulk. In addition to the exciting possibilities for application, from the fundamental point of view, ferromagnetic NWs will provide an opportunity to investigate the spin-Seebeck effect in reduced dimensions [15]. A deeper understanding of how spins and phonons couple thermodynamically could in turn lead to fundamentally new applications, such as spin-based cooling and magnetically sensitive thermoelectrics.

Here, we investigate the thermoelectric properties of $\mathrm{Ga}_{0.95} \mathrm{Mn}_{0.05}$ As NWs. Combining thermopower and conductance (or resistance) measurements can provide information on carrier density when conventional characterization techniques via the Hall effect and field effect are not possible [16]. We were able to estimate the hole carrier density from thermopower measurements to be $p \sim 10^{17}-10^{18} \mathrm{~cm}^{-3}$ in our NW. In addition, we find a dramatic rise in the resistance and thermopower of the NW below $120 \mathrm{~K}$ [17-19]. The resistance versus temperature measurements point to the role of Mott variable range hopping $(\mathrm{VRH})$ transport with activation energy $62 \mathrm{meV}$ at $100 \mathrm{~K}$ and hopping lengths of $11 \mathrm{~nm}$. We show that the addition of a term due to Mott variable range hopping $(\mathrm{VRH})$ transport [20-22] can also 
qualitatively describe the rise in thermopower observed in these $\mathrm{Ga}_{0.95} \mathrm{Mn}_{0.05}$ As NWs. However, our model suggests that a simple parabolic-band picture is not fully adequate, implying that more complex behavior is taking place.

\section{Experimental Methods}

GaAs NWs of $40 \mathrm{~nm}$ diameter were grown by MOVPE and subsequently implanted with $\mathrm{Mn}$ ions to doping concentrations of 0.5 to $2.9 \%$, corresponding to $\mathrm{Ga}_{1-x} \mathrm{Mn}_{x}$ As with $x=0.01$ to 0.058 stoichiometry. Both simulations and transmission electron microscopy (TEM) showed that the NWs are of high crystalline quality after implantation and that the $\mathrm{Mn}$ is reasonably homogeneously distributed. In this work, we concentrate on the higher doped wires. The NW growth and implantation techniques were discussed in detail previously [13].

To prepare devices for thermoelectric characterization, NWs were collected from the growth substrate with cleanroom tissue paper and then brushed onto $\mathrm{Si} / \mathrm{SiO}_{2}$ chips with $110 \mathrm{~nm}$ thick oxide. A thick metallic plane was evaporated on the backside of the chip for voltage-gating measurements. We searched optically for suitable NWs for processing and determined the location of the NWs relative to predefined $\mathrm{Au}$ alignment marks on the $\mathrm{SiO}_{2}$ chips. Contacts to the NWs were fabricated via standard e-beam lithography processes. Briefly, 950-A5 PMMA was spun onto the sample at $5000 \mathrm{rpm}$ for $60 \mathrm{~s}$ and baked at $180^{\circ} \mathrm{C}$ for 5 minutes. After the contact pattern exposure, the resistance was developed in MIBK: IPA = 1:3 for $30 \mathrm{~s}$. To ensure good ohmic contacts to the NWs, we performed the following procedures: the samples were treated in $\mathrm{HCl} / \mathrm{H}_{2} \mathrm{O}$ solution for 15 seconds followed by passivation in $\left(\mathrm{NH}_{4}\right)_{2} \mathrm{~S}_{x}$ solution at $40^{\circ} \mathrm{C}$ for 2 minutes. Then metal contacts of $\mathrm{Pd}(10 \mathrm{~nm}) / \mathrm{Zn}$ $(10 \mathrm{~nm}) / \mathrm{Pd}(35 \mathrm{~nm})$ were evaporated followed by lift-off [23]. Measurements were done in a Janis Varitemp cryostat between $60 \mathrm{~K}$ and $190 \mathrm{~K}$.

\section{Results and Discussions}

Figure 1 shows a scanning electron microscope (SEM) image of a typical device similar to the one measured. Current is driven through an on-chip metallic microstrip line to generate a temperature differential $\Delta T$ along the length of the NW. Metallic contacts to the NWs can be used to probe in separate measurements the voltage drop or conductance. The metal contacts also serve as thermometers, where we calibrate the four-probe resistance change of the metal against the cryostat temperature $T$; see thermometer I and II in Figure 1. The change in the resistance of the metallic thermometers as the cryostat temperature is changed allows for the determination of the temperature at the metal to nanowire contact point to within $0.1 \mathrm{~K}$. Similar device geometries with patterned microstrip line heaters and thermometers have been used to study thermopower in, for example, carbon nanotubes $[24,25]$ and other materials [26]. We typically measured in the linear response regime, maintaining $\Delta T \ll T$. For the substrate temperatures shown,

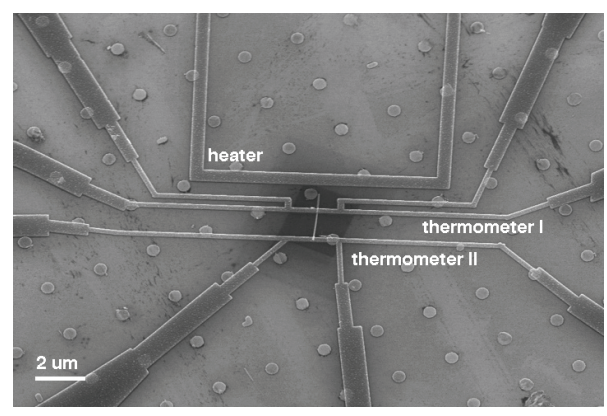

Figure 1: Scanning electron microscope image of a typical nanowire device with heater and thermometer metallic microstrip lines is shown. In a separate measurement, the contact electrodes can be independently used to probe the voltage drop along the nanowire or conductance. Image is of a device similar to the one that is measured.

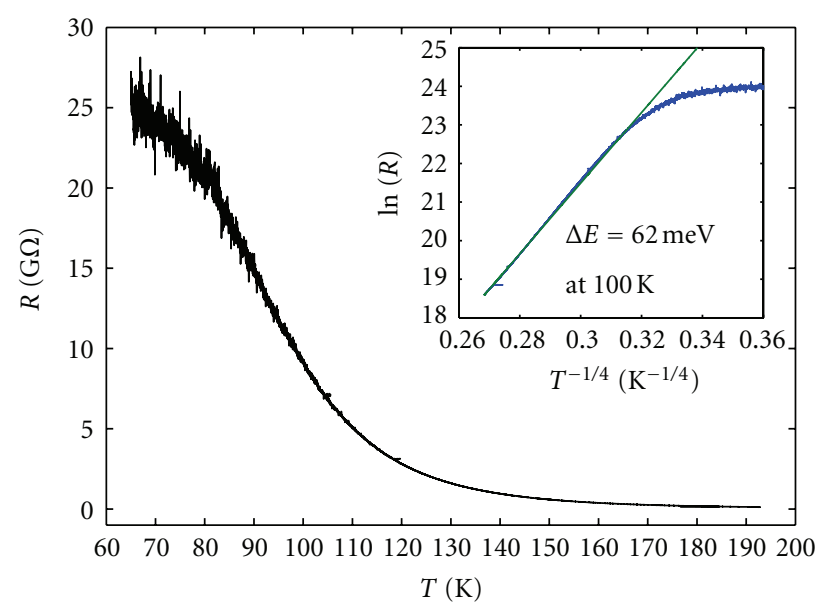

FIGURE 2: $\mathrm{Ga}_{0.95} \mathrm{Mn}_{0.05}$ As nanowire resistance as a function of temperature. The inset shows a plot of $\ln (R)$ versus $T^{-1 / 4}$, with the extracted activation energy of $62 \mathrm{meV}$ at $100 \mathrm{~K}$.

the gradient generated along the wire was typically $\Delta T \sim 1 \mathrm{~K}$. A metallic backgate was also available for field effect, but we found that the NW transport did not respond significantly to the applied gate voltages.

We discuss first the electronic transport of the NW as a function of temperature. In Figure 2, we show the resistance calculated from the measured two terminal conductances. The contact resistances obtained from our fabrication procedure were found to be from $500 \Omega$ to $1 \mathrm{k} \Omega$ (for wires with 4 terminal contacts), and we conclude that the measured response is due to the wire itself [27]. The NWs show dramatic increase in the resistance for $T<120 \mathrm{~K}$, and this rise saturates below $T \sim 80-90 \mathrm{~K}$. In order to obtain a better understanding of the transport mechanisms down to $60 \mathrm{~K}$, we plot $\ln (R)$ versus $T^{-1 / 4}$ in the inset of Figure 2 . The linearity of such a trace indicates that Mott variable range hopping may likely be in play [20,27]. Deviation from linearity occurs around $T^{-1 / 4}=0.32$ or $T \sim 95 \mathrm{~K}$. From the slope of this trace, we estimate the carrier density to be approximately $3 * 10^{18} \mathrm{~cm}^{-3}$ at $100 \mathrm{~K}$ with hopping lengths 
of $11 \mathrm{~nm}$. This allows a determination of an activation energy of $\Delta E=62 \mathrm{meV}$ at $100 \mathrm{~K}$. For larger $80 \mathrm{~nm}$ diameter wires, similar behavior was found though the hopping transport was found to persist down to $T \sim 50 \mathrm{~K}$ [27].

Figure 3 shows the measured thermopower, which is defined as $S=-\Delta V / \Delta T$, where $\Delta V$ is the measured voltage drop across the nanowire. The positive sign of the measured thermopower indicates that the transport carriers are holes. This is expected for Mn-doped GaAs since substitutional Mn is an acceptor in GaAs. The thermopower is seen to increase dramatically for temperatures lower than $120 \mathrm{~K}$, which is the temperature where the electrical resistance also begins to sharply increase. In order to understand why the thermopower rises to the rather large values of $\sim 800 \mu \mathrm{V} / \mathrm{K}$, we first consider the thermopower in the paramagnetic regime (without taking into account hopping transport) consisting of the following two terms:

$$
S_{\text {tot }}=S_{\text {diff }}+S_{\text {exch }}
$$

The first term is essentially the semiclassical Mott relation for diffusive transport [16],

$$
S=-\left.\frac{\pi^{2} k_{B}^{2} T}{3|e|} \frac{d \ln \sigma}{d E}\right|_{E=E_{F}}=-\frac{\pi^{2} k_{B}^{2} m^{*}}{\left(3 \pi^{2}\right)^{2 / 3} \hbar^{2}|e|} \frac{T}{p^{2 / 3}} .
$$

Here, $\sigma$ is the conductivity, $k_{B}$ is the Boltzmann constant, $e$ is the electron charge, $\hbar$ is Planck's constant, and $p$ is the carrier density. The hole effective mass for GaMnAs is taken to be $m^{*}=0.5 * m_{e}$, where $m_{e}$ is the free electron mass [28]. The second term is from exchange mechanisms

$$
\begin{gathered}
S_{\text {exch }}=\frac{S_{0} T}{T+T_{0}}, \\
S_{0}=\frac{4 \pi^{2} k_{B}}{e} D\left(E_{F}\right) I_{p d} V \frac{\rho_{\text {exch }}}{\rho},
\end{gathered}
$$

where $D\left(E_{F}\right)$ is the density of states at the Fermi energy, $I_{p d}$ is the exchange integral between carriers and magnetic centers, $V$ is the nonmagnetic scattering potential, and $\rho_{\operatorname{exch}} / \rho$ is the ratio between exchange contribution to resistivity to total resistivity. Here, $T_{0}$ is a material-dependent parameter with weak temperature and magnetic impurity dependence, and for thin films it is found to be $T_{0}=150 \mathrm{~K}$ [21]. We utilize these functional forms to obtain a fit to the data above $120 \mathrm{~K}$, where the thermopower is relatively flat, as seen in Figure 3 (blue dot dash curve). We find that the parameters that give the best fit to the curve are $p \sim 3 * 10^{17} \mathrm{~cm}^{-3}$ and $S_{0} \sim 4 \mathrm{mV} / \mathrm{K}$, with $T_{0}=150 \mathrm{~K}$ fixed based on the thin film value. Due to the limited number of data points, we believe our estimate for the density to be correct to within an order of magnitude. This is comparable to what has been observed for comparable nanowires [27] and is, within our accuracy, consistent with the estimate obtained from the resistance versus temperature curves above. Note that the value of $S_{0}$ is unusually large, a point that we will return to below. These results demonstrate that thermopower presents a powerful method for extracting carrier sign and density when conventional Hall effect and field effect techniques are not possible.

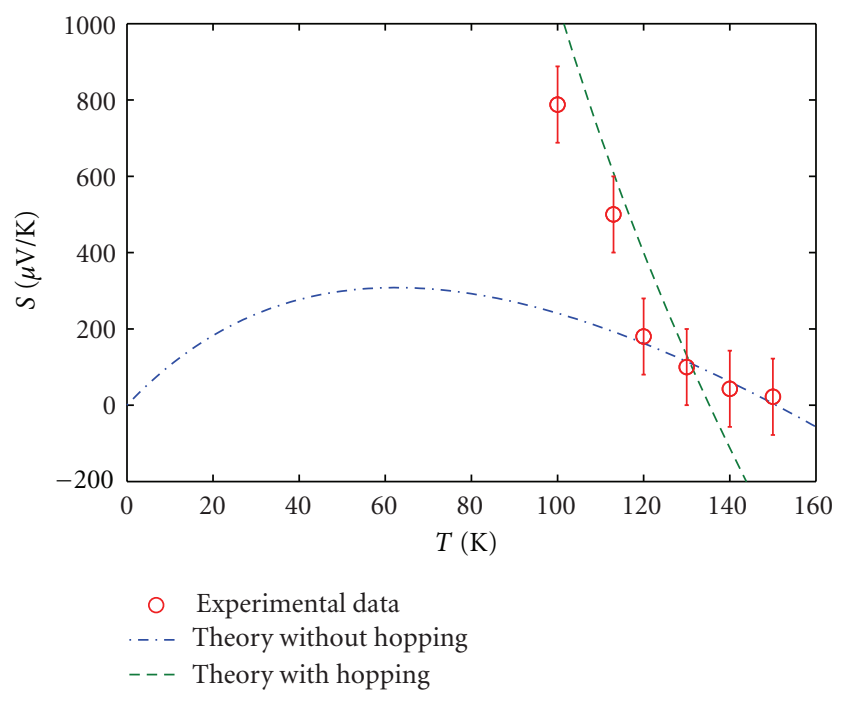

Figure 3: Thermopower for the $\mathrm{Ga}_{0.95} \mathrm{Mn}_{0.05}$ As nanowire device as function of temperature. The experimental data matches well at high temperature with values calculated using a model without hopping [21]. However, at temperatures below $120 \mathrm{~K}$, the nanowire thermopower starts to rise far above the extrapolation. Addition of a hopping term qualitatively accounts for the rise in thermopower.

Below $120 \mathrm{~K}$, it is clear that additional contributions to the thermopower must be considered to explain the observed data. Taking a cue from the temperature dependence of the resistance, we consider adding a term due to hopping conduction. We see below that this hopping contribution, $S_{\text {hop, }}$ can qualitatively describe the observed experimental behavior. We start with a hopping term of the form [21]

$$
S_{\mathrm{hop}}=\left(F_{\mathrm{corr}} * \frac{k_{B}}{e}\right)\left(\frac{\Delta E}{k_{B} T}+A\right),
$$

where $A$ does not have significant temperature dependence and includes an additional factor, $F_{\text {corr }}$, to account for electron correlations. Without $F_{\text {corr }}$, $(4)$ expresses that, in the simple case of single particle, noninteracting transport, the thermopower of a device or material essentially measures the average energy (here assumed to be the activation energy $\Delta E$ ) where transport takes place. By plotting the experimental data versus $1 / T$, and taking the correlation factor, $F_{\text {corr }} \sim 5$, we can extract the activation energy $\Delta E \sim$ $63 \mathrm{meV}$, in good agreement with that seen in the $R(T)$, as seen in Figure 4. Based on this, we can calculate the full theoretical trace including hopping mechanisms as shown in Figure 3 (green-dashed line). We see that inclusion of this hopping mechanism can describe the observed rise in thermopower. At $100 \mathrm{~K}$, the theoretical trace seems to overshoot the experimental data. This is consistent with the fact that the $\ln (R)$ versus $T^{-1 / 4}$ trace starts to deviate from linearity around $95 \mathrm{~K}$.

Comparing the results from thermopower to that obtained from the electrical resistance, we see that an activation energy of $62 \mathrm{meV}$ at $100 \mathrm{~K}$ is deduced from Mott VRH theory [27]. In order for (4) to give a comparable extracted 


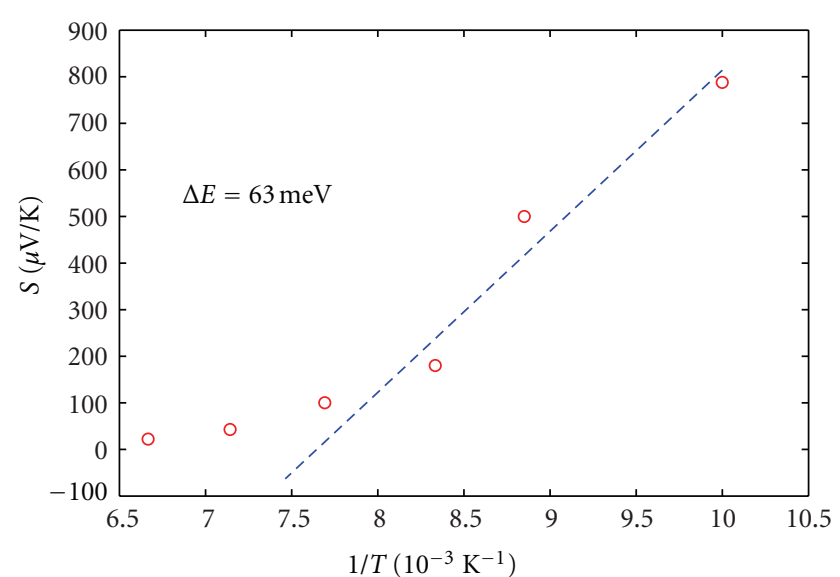

FIgURE 4: Extracted hopping activation energy, based on a hopping term $S_{\text {hop }} \sim \Delta E / T$. Further discussion can be found in the text.

activation energy, we required $F_{\text {corr }} \sim 5$. This implies strongly that correlation effects play a more significant role here. The fact that $F_{\text {corr }}$ is much larger than one is not so surprising if we consider the unusually large measured thermopower. Indeed, colossal thermopowers have been seen in strongly correlated semiconductors such as FeSb [29], where the large thermopowers are presumed to be a result of the Fe 3d-states and Sb 5p-states hybridizing into a coherent, high mobility state. We note that compared to 2D GaMnAs films, which exhibit hole-mediated ferromagnetic coupling, these GaMnAs nanowires are clearly in the hopping regime, with a relatively low hole concentration and large hopping lengths. These observations are an indication of acceptor compensation from $\mathrm{Mn}$ interstitial impurities and As antisites, which were created by the ion beam implantation process. More analysis will be necessary to understand the relative importance of these two contributions. Clearly then, our results (i) highlight the important role that thermopower measurements can play as a characterization tool complementary to conductance measurements, and (ii) that our heavily Mn-doped nanowires exhibit more complex electronic behavior than is apparent from conductance measurements, leaving much room for further investigation.

\section{Conclusions}

In this work, we studied the thermopower and conductance of $\mathrm{Ga}_{0.95} \mathrm{Mn}_{0.05}$ As nanowires. We utilize thermopower measurements as a means to estimate the hole carrier concentration when Hall effect and field effect are not possible. We find from thermopower and from conductance measurements the hole density $p \sim 10^{17}-10^{18} \mathrm{~cm}^{-3}$. Furthermore, we observe dramatic increases in both thermopower and resistance at low temperature. The resistance versus temperature suggests that the NW is in the regime of variable range hopping transport. From the thermopower, we deduce that additional correlated phenomena must be taking place. These fundamental studies provide critical insight into the transport mechanisms likely to be found in next-generation GaMnAs and ferromagnetic NWs.

\section{Acknowledgments}

The authors acknowledge support from nmC@LU, Swedish Energy Council (Energimyndigheten) Grant no. 32920-1, the Swedish Research Council, the Strategic Foundation, and Knut and Alice Wallenberg Foundation. One of the authors, W. Paschoal Jr., gratefully acknowledges financial support from the Pará Education Secretary (SEDUC) and the Pará Government School (EGPA) of Brazil.

\section{References}

[1] Y. Cui, Z. Zhong, D. Wang, W. U. Wang, and C. M. Lieber, "High performance silicon nanowire field effect transistors," Nano Letters, vol. 3, no. 2, pp. 149-152, 2003.

[2] P. H. Yeh, Z. Li, and Z. L. Wang, "Schottky-gated probe-free $\mathrm{ZnO}$ nanowire biosensor," Advanced Materials, vol. 21, no. 48, pp. 4975-4978, 2009.

[3] A. I. Hochbaum and P. Yang, "Semiconductor nanowires for energy conversion," Chemical Reviews, vol. 110, no. 1, pp. 527$546,2010$.

[4] J. Sadowski, P. Dłuzewski, S. Kret et al., "GaAs:Mn nanowires grown by molecular beam epitaxy of (Ga,Mn)As at MnAs segregation conditions," Nano Letters, vol. 7, no. 9, pp. 27242728, 2007.

[5] F. Martelli, S. Rubini, M. Piccing et al., "Manganese-induced growth of GaAs nanowires," Nano Letters, vol. 6, no. 9, pp. 2130-2134, 2006.

[6] M. F. H. Wolff, D. Görlitz, K. Nielsch, M. E. Messing, and K. Deppert, "Synthesis and magnetic characterization of MnAs nanoparticles via nanoparticle conversion," Nanotechnology, vol. 22, no. 5, Article ID 055602, 2011.

[7] J. Adell, I. Ulfat, J. Sadowski, L. Ilver, and J. Kanski, "Electron spectroscopic studies of nanowires formed by (GaMn)As growth on GaAs(111)B," Solid State Communications, vol. 151, no. 11, pp. 850-854, 2011.

[8] H. C. Jeon, T. W. Kang, T. W. Kim, Y. J. Yu, W. Jhe, and S. A. Song, "Magnetic and optical properties of (Ga1-X $\mathrm{Mnx}$ ) As diluted magnetic semiconductor quantum wires with above room ferromagnetic transition temperature," Journal of Applied Physics, vol. 101, no. 2, Article ID 023508, 2007.

[9] A. Rudolph, M. Soda, M. Kiessling et al., "Ferromagnetic GaAs/GaMnAs core-shell nanoWires Grown by Molecular Beam Epitaxy," Nano Letters, vol. 9, no. 11, pp. 3860-3866, 2009.

[10] H. S. Kim, Y. J. Cho, K. J. Kong et al., "Room-temperature ferromagnetic Ga1-x-MnxAs ( $\mathrm{X}<=0.05)$ nanowires: dependence of electronic structures and magnetic properties on $\mathrm{Mn}$ content," Chemistry of Materials, vol. 21, no. 6, pp. 1137-1143, 2009.

[11] P. Dluzewski, J. Sadowski, S. Kret, J. Dabrowski, and K. Sobczak, "TEM determination of directions of (Ga,Mn)As nanowires grown by $\mathrm{MBE}$ on $\mathrm{GaAs}(001)$ substrates," Journal of Microscopy, vol. 236, no. 2, pp. 115-118, 2009.

[12] D. D. Awschalom, N. Samarth, and D. Loss, Semiconductor Spintronics and Quantum Computation, Springer-Verlag, Heidelberg, Germany, 2002. 
[13] C. Borschel, M.E. Messing, M.T. Borgstrom et al., "A new route toward semiconductor nanospintronics: highly $\mathrm{Mn}$ doped GaAs nanowires realized by ion-implantation under dynamic annealing conditions," Nano Letters, vol. 11, no. 9, pp. 3935-3940, 2011.

[14] L. Chen, X. Yang, F. Yang et al., "Enhancing the curie temperature of ferromagnetic semiconductor (Ga,Mn)As to 200 K via nanostructure engineering," Nano Letters, vol. 11, no. 7, pp. 2584-2589, 2011.

[15] Y. Dubi and M. Di Ventra, "Thermospin effects in a quantum dot connected to ferromagnetic leads," Physical Review B, vol. 79, no. 8, Article ID 081302, 2009.

[16] C. H. Lee, G. C. Yi, Y. M. Zuev, and P. Kim, “Thermoelectric power measurements of wide band gap semiconducting nanowires," Applied Physics Letters, vol. 94, no. 2, Article ID 022106, 2009.

[17] Y. I. Ravich and S. A. Nemov, "Hopping conduction via strongly localized impurity states of indium in $\mathrm{PbTe}$ and its solid solutions," Semiconductors, vol. 36, no. 1, pp. 1-20, 2002.

[18] O. E. Parfenov and F. A. Shklyaruk, "On the temperature dependence of the thermoelectric power in disordered semiconductors," Semiconductors, vol. 41, no. 9, pp. 1021-1026, 2007.

[19] D. Gitsu, T. Huber, L. Konopko, and A. Nikolaeva, "Peculiarities of thermopower in Bi microwires at low temperatures," Physica Status Solidi B, vol. 242, no. 12, pp. 2497-2502, 2005.

[20] N. F. Mott and E. A. Davis, Electronic Processes in NonCrystalline Materials, Clarendon Press, Oxford, UK, 1979.

[21] V. Osinniy, K. Dybko, A. Jedrzejczak et al., “Thermoelectric studies of electronic properties of ferromagnetic GaMnAs layers," Semiconductor Physics, Quantum Electronics and Optoelectronics, vol. 11, no. 2, pp. 257-265, 2008.

[22] B. L. Sheu, R. C. Myers, J. M. Tang et al., "Onset of ferromagnetism in low-doped Ga1-xMnxAs," Physical Review Letters, vol. 99, no. 22, Article ID 227205, 2007.

[23] J. Wallentin, J. M. Persson, J. B. Wagner, L. Samuelson, K. Deppert, and M. T. Borgström, "High-performance single nanowire tunnel diodes," Nano Letters, vol. 10, no. 3, pp. 974979, 2010.

[24] J.P. Small, K.M. Perez, and P. Kim, "Modulation of thermoelectric power of individual carbon nanotubes," Physical Review Letters, vol. 91, no. 25, pp. 2568011-2568014, 2003.

[25] M. C. Llaguno, J. E. Fischer, A. T. Johnson, and J. Hone, "Observation of thermopower oscillations in the coulomb blockade regime in a semiconducting carbon nanotube," Nano Letters, vol. 4, no. 1, pp. 45-49, 2004.

[26] W. Liang, A. I. Hochbaum, M. Fardy, O. Rabin, M. Zhang, and P. Yang, "Field-effect modulation of seebeck coefficient in single PbSe nanowires," Nano Letters, vol. 9, no. 4, pp. 1689 1693, 2009.

[27] W. Paschoal Jr, S. Kumar, C. Borschel et al., "Hopping conduction in Mn ion implanted GaAs Nanowires," Nano Letters, vol. 12, no. 9, pp. 4838-4842, 2012.

[28] H. Ohno, "Making nonmagnetic semiconductors ferromagnetic," Science, vol. 281, no. 5379, pp. 951-956, 1998.

[29] A. Bentien, S. Johnsen, G. K. H. Madsen, B. B. Iversen, and F. Steglich, "Colossal seebeck coefficient in strongly correlated semiconductor $\mathrm{FeSb}_{2}$," Europhysics Letters, vol. 80, no. 1, Article ID 17008, 2007. 

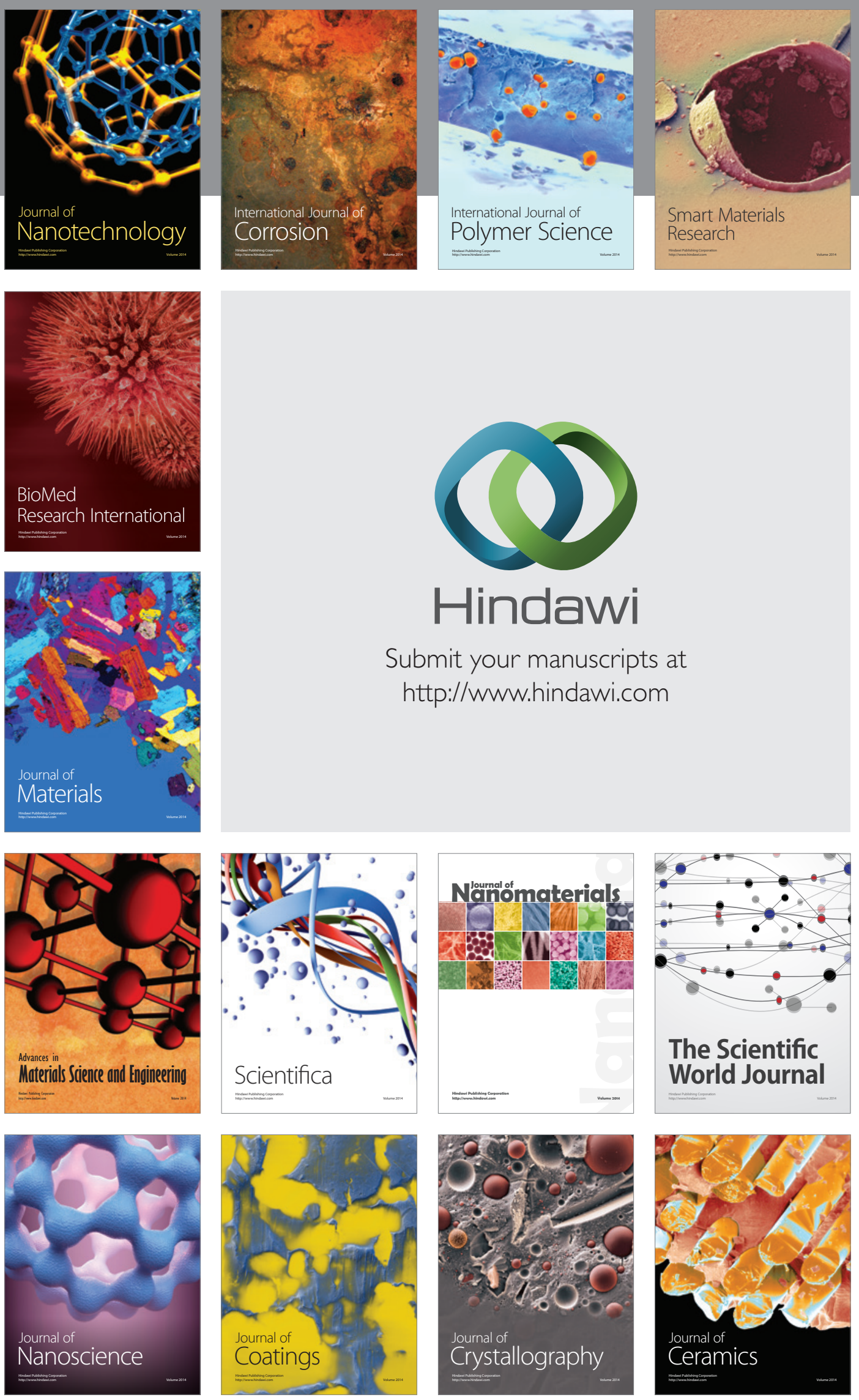

The Scientific World Journal

Submit your manuscripts at

http://www.hindawi.com

\section{World Journal}

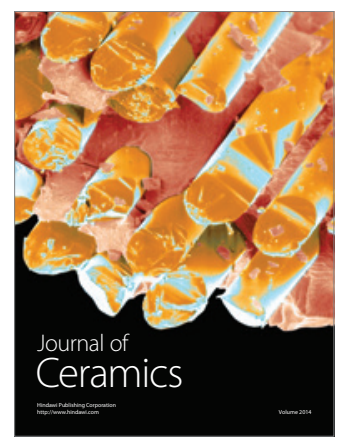

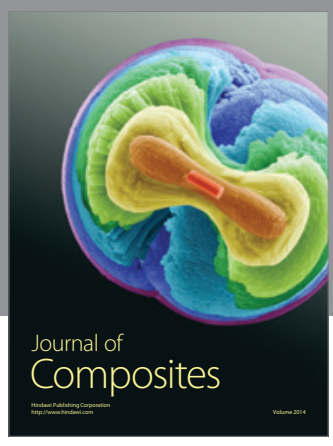
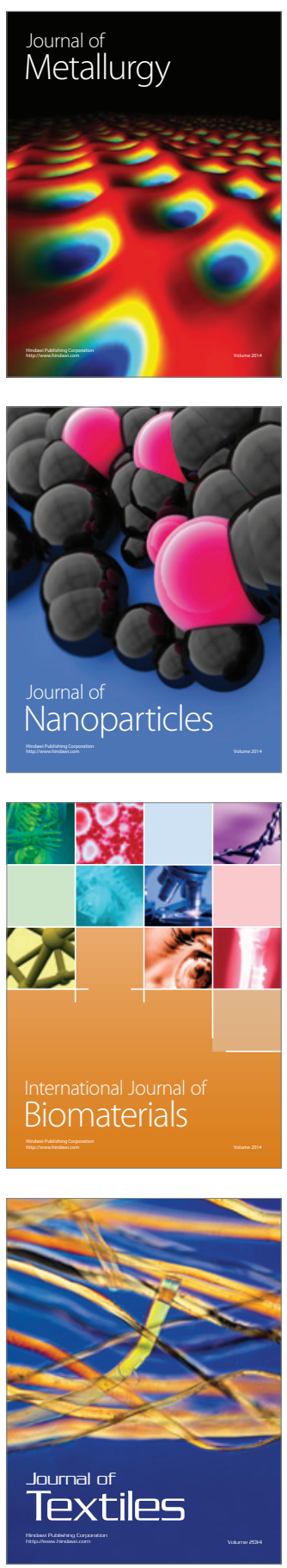